\title{
Estimating domains of attraction of fuzzy polynomial systems
}

\author{
Pitarch, J.L. ${ }^{1}$ Ariño, C.V. ${ }^{2}$ Sala, A. ${ }^{3}$ \\ ${ }^{1,3}$ Universidad Politecnica de Valencia, Valencia, Spain. \\ ${ }^{2}$ Universitat Jaume I, Castello de la Plana, Spain.
}

\begin{abstract}
Most fuzzy control papers check LMI or SOS stability conditions in order to prove local stability results of nonlinear systems (Takagi-Sugeno fuzzy models or polynomial fuzzy models via Sector Nonlinearity approach). In case of having into account the shape of the membership functions in a particular region of interest, less conservative stability and stabilization conditions can be easily set up. In this paper local polynomial stability results are explored in order to obtain the largest basin of attraction for a particular system.
\end{abstract}

Keywords: Lyapunov function, basin of attraction estimation, fuzzy systems, Takagi-Sugeno models, polynomial systems, virtual membership functions, polynomial stability

\section{Introduction}

The sector-nonlinearity approach allows obtaining locally exact fuzzy models from smooth nonlinear systems [1]. The idea has been recently extended to fuzzy polynomial systems [2].

Once such local models are available, fuzzy stability analysis and control design techniques can be explored on them. Currently, many papers approach fuzzy control via LMI (Linear Matrix Inequality) techniques, discussed in [3] in a classic control context and introduced in, for instance, $[1,4]$ in the fuzzy systems community, or SOS (Sum of Squares) techniques introduced recently in [5] and discussed in $[6]$.

In most literature contributions, LMI and SOS stability conditions are devised in order to prove global stability and performance of Takagi-Sugeno [7] fuzzy systems; however, such laws are usually independent of the values of membership functions, and fulfill for any arbitrary shapes of them. Knowledge of the shape of the membership functions may allow to lift some conservativeness. Some constraints may hold globally, and some others only locally.

Somehow, the original nonlinear control problem is forgotten in most of the results. Assuming that global stability of the nonlinear system is not feasible, the objective of the analysis or controller de- sign phases should be obtaining the largest region in which a particular performance bound is attained.

For instance, Example 6 in [1](Chapter 2) shows that the basin of attraction for fuzzy systems may be membership dependent: the same vertices but different memberships give rise to different (nonlinear) basins of attraction. Anyway, unless fuzzyLyapunov functions are used $[8,9]$, the basins of attraction proved with LMIs are not membership dependent. In many fuzzy control results, once LMIs or SOS conditions are set up and found feasible, a stabilising solution is said to be found. However, although a decreasing Lyapunov function $V(x)$ has been found, local stability in $\Omega$ has not been proved, but only in the largest invariant set $V_{\gamma}=\{x \mid V(x) \leq \gamma\}$ such that $V_{\gamma} \subset \Omega$. Illconditioned Lyapunov functions may be of little use for practical results.

The basic idea in this paper is exploring, once a local fuzzy polynomial model of a nonlinear system is obtained, how to obtain local results in a smaller region of interest without remodelling. In [10], an expression of the membership functions as a convex combination of other ones allows recasting the original model.

In this paper, the idea is applied to stability analysis (could be applied to controller design) and it is shown that ensuring the largest spherical basin of attraction around the origin requires an iterative procedure, as intuitively expected. The idea applies to sophisticated Lyapunov functions or performance inequalities or to non-spherical (polytopic) target shapes.

In particular, in this paper local polynomial stability results are obtained, even in the case global polynomial-stability related SOS conditions are infeasible. As long as the Jacobian linearisation at the origin is (strictly) stable, a local result will be obtained with a polynomial Lyapunov function for a small enough region of interest. If the Jacobian were not strictly stable, the method may fail (as stability of the nonlinear system is not guaranteed in this case, nor the existence of a local Lyapunov function).

The structure of the paper is as follows: Next section discusses notation and widely-known stability results. Section 3 discusses a transformation of 
a fuzzy model when the membership functions are themselves a convex combination of some vertices (and proves that it is the case when the maximum and minimum of them in a particular region are known). Section 4 applies the results to find the largest local polynomial stable region and proposes an iterative algorithm. One example is provided in Section 5 and a conclusion section summarises the main results.

\section{Preliminaries}

A polynomial fuzzy system is a system whose dynamics can be expressed as

$$
\dot{x}_{i}=p_{i}(x, u, z, \mu), \quad i=1, \ldots, n
$$

where $p_{i}(x, u, z, \mu)$ is a polynomial in the variables $x, u, z, \mu$ ( $x$ is the state, $u$ the control action, $\mu$ the membership functions and $z$ some funtions of time) such that $p_{i}(0,0,0, \mu)=0 \forall \mu$. Let us consider a Takagi-Sugeno [7] (TS) fuzzy model where fuzzy IF-THEN rules represent local polynomial dynamics (via Taylor series approach [2]) of a nonlinear system. The rules of the TS fuzzy models are usually expressed as:

\section{IF $z$ is $M_{i}$ THEN}

$$
\begin{array}{r}
\dot{x}=p_{i}(z)+B_{i}(z) u \\
y=C_{i}(z)
\end{array}
$$

The local polynomial models represented by $p_{i}(z)+$ $B_{i}(z) u$ are used to compute the final output as follows:

$$
\dot{x}=\sum_{i=1}^{r} \mu_{i}(z)\left(p_{i}(z)+B_{i}(z) u\right)
$$

where $\mu_{i}$ represents the membership functions of fuzzy set $M_{i}$ such that:

$$
\sum_{i=1}^{n} \mu_{i}(z)=1, \quad \mu_{i}(z)>0 \forall z i: 1 \ldots n
$$

\subsection{Stability of fuzzy polynomial systems}

Lyapunov stability theory proves that a system expressed as (1) without control action $(u=0)$ is stable if exist a function $V(x)$ such that:

$$
V(x)>0, \frac{d V}{d x}<0, V(0)=0, \forall x
$$

Some cases in Lyapunov stability analysis of TS fuzzy systems may be approached as a linear matrix inequality (LMI) optimization problem [3]. The most popular Lyapunov Functions proposed in literature are quadratic forms: $V(x)=x^{T} P x$. This type of Lyapunov functions fulfill the stability conditions if $P$ is a definite positive matrix and

$$
A_{i}^{T} P+P A_{i}<0, i: 1 . . n
$$

The above $n$ equations are LMI's, hence widely avaliable LMI optimization software either finds a $P$ or determines that the LMI is infeasible. The reader is referred to [1] for ample discussion.

Now consider a polynomial fuzzy system that is expressed as (1). For operational purposes [11], the polynomials should be made homogeneous in the membership functions $\mu$, which are positive, and it will be described by the change of variable $\mu_{i}=\sigma_{i}^{2}$, which results in a model $\dot{x}_{i}=\tilde{p}_{i}(x, u, z, \sigma)$. In addition, for only stability analysis and without presence of external disturbances or whatever $z$, the model of study will be $\dot{x}_{i}=\tilde{p}_{i}(x, \sigma)$.

Stability will be proved if a polynomial Lyapunov function $V(x)$ is found to verify

$$
\begin{aligned}
V(x)-\epsilon & \in \Sigma_{x} \\
R(\sigma, x):=-\frac{d V}{d x} \tilde{p}(x, \sigma) & \in \Sigma_{x, \sigma}
\end{aligned}
$$

where $\epsilon$ is a radially unbounded positive polynomial, which usually is $\sum x_{i}^{2}$ (but not necessary so), and $\Sigma$ means SOS.

Indeed, setting $V(x)$ to be an arbitrary degree polynomial in the state variables, $\dot{V}(x)$ is also a vector of polynomials in the variables $x$ and $\sigma$. Hence, $\dot{V}(x)$, which is denoted as $R(\sigma, x)$ in (6), is a polynomial. If $V(x)$ is linear in some decision variables (the natural choice is the polynomial coefficients), also it's $\dot{V}(x)$, and expressions (5) and (6), can be directly introduced into SOS programming packages $[12,13]$ in order to get values of the decision variables that fulfill the earlier constraints. The key idea in the SOS approach is that an even-degree polynomial $p(a)$ is SOS if and only if there exists a vector of monomials $m(a)$ and a constant positive-definite matrix $H$ such that $p(a)=m(a)^{T} H m(a)$; this way, SOS problems can be solved via semidefinite-programming tools, which search for such an $H$. See [14] for details. Evidently, all SOS polynomials are positive, but the converse is not true [16].

Examples of stability analysis of such systems have been presented in $[11,15]$ and [2].

Remark: Note that the membership functions $\mu$ do not appear in the LMI conditions. Hence,for instance, the same $P$ defines a quadratic Lyapunov function for multiple nonlinear systems with the same "vertex models" as the original one. Such generality is good in case a feasible $P$ is found but, on the contrary, it is a too restrictive condition that in some cases results in infeasibility being the underlying system actually stable.

\subsection{Local stability of fuzzy polynomial systems}

When the above LMI/SOS problems are unfeasible, other alternative conditions must be sought. Piecewise Lyapunov functions are discussed in [16], Fuzzy 
Lyapunov functions are discussed in [17], non-PDC regulator are discussed in [9], etc.

A different alternative, not commonly explored in current literature, in the authors' opinion, is trying to achieve local stability results in a zone around the equilibrium as large as possible. Such a result is motivated on the first Lyapunov theorem for local stability: if the linearised system in $x=0$ is exponentially stable, then so it is the nonlinear one, for initial conditions in a sufficiently small neighborhood of $x=0$. Polynomial characterisations of subsets of those basins of attraction may be expressed via LMI/SOS conditions, as discussed in the sequel.

The objective of this paper is to try to reach better results in local stability in a prefixed-shape zone arround equilibrium as large as possible. A convex subset of the original model in a small region of interest is used in order to reduce conservativeness.

\section{Local Fuzzy Polynomial Models}

In order to analyze the local stability of a TS fuzzy model or fuzzy polynomial model (3) within a region, the original model is modified using the information of the membership functions.

The idea is to express the the membership functions $\mu(x)$ of a fuzzy system, valid in a region $\Omega$, as a convex sum of some vectors $v_{k}$. Proof can be checked in [18].

In case of fuzzy polynomial models, same idea can be aplied:

Lemma 1 If the membership functions $\mu(z)$ of a fuzzy polynomial system described as (3) in a region $\Omega$, can be themselves expressed as a convex sum of some vectors $v_{k}$ :

$$
\mu(z)=\sum_{k=1}^{n_{v}} \beta_{k}(z) v_{k}, \quad \forall z \in \Omega
$$

where:

$$
\begin{gathered}
\mu(z)=\left[\mu_{1}(z), \mu_{2}(z), \ldots, \mu_{n}(z)\right] \\
\sum_{k=1}^{n_{v}} \beta_{k}(z)=1, \beta_{k}(z)>0 \forall z \in \Omega
\end{gathered}
$$

Then, for polynomial fuzzy models, the system can be transformed to:

$$
\dot{x}=\sum_{k=1}^{n_{v}} \beta_{k}(z) p_{k}(Z)^{*}
$$

where

$$
p_{k}(Z)^{*}=\sum_{i}^{n} v_{k i} p_{i}(Z)
$$

and $Z$ is a vector of monomials of $x$.
Proof: The expression (7) can be substituted in the system equation (3):

$$
\begin{aligned}
\mu(z) & =\sum_{k=1}^{n_{v}} \beta_{k}(z) v_{k} \\
v_{k} & =\left[v_{k 1}, v_{k 2}, \ldots, v_{k n}\right] \\
\mu_{i}(z) & =\sum_{k=1}^{n_{v}} \beta_{k}(z) v_{k i} \\
\dot{x} & =\sum_{i=1}^{n} \sum_{k=1}^{n_{v}} \beta_{k}(z) v_{k i} p_{i}(Z) \\
\dot{x} & =\sum_{k=1}^{n_{v}} \beta_{k}(z) \sum_{i}^{n} v_{k i} p_{i}(Z)
\end{aligned}
$$

so the local representation of the system in $\Omega$

$$
\dot{x}=\sum_{k=1}^{n_{v}} \beta_{k}(z) p_{k}(Z)^{*} \quad \forall z \in \Omega
$$

where:

$$
\sum_{k=1}^{n_{v}} \beta_{k}(z)=1 \beta_{k}(z)>0 \forall z \in \Omega k: 1 \ldots n_{v}
$$

The convex-combination conditions for the membership functions required in the above lemmas are easy to meet. Indeed $\mu_{i}$ are assumed known in fuzzy systems. Then, the result below may be applied to obtain a (possibly conservative) vertex set.

Note 1 Let us consider a region $\Omega$. If bounds $\mu_{i}^{M}$ and $\mu_{i}^{m}$ on the extremum values of the membership functions in $\Omega$ can be computed, in such a way that:

$$
\mu_{i}^{M} \geq \max _{z \in \Omega} \mu_{i}(z) \quad \mu_{i}^{m} \leq \min _{z \in \Omega} \mu_{i}(z)
$$

then there exist a set of $\beta_{k}(z), k=1, \ldots, n_{v}$ so that the vector of membership functions

$$
\mu(z)=\left[\mu_{1}(z), \mu_{2}(z), \ldots, \mu_{n}(z)\right]
$$

may be expressed in $\Omega$ as:

$$
\mu(z)=\sum_{k=1}^{n_{v}} \beta_{k}(z) v_{k}, \quad z \in \Omega
$$

where:

$$
\sum_{k=1}^{n_{v}} \beta_{k}(z)=1, \beta_{k}(z)>0 \forall z \in \Omega
$$

Indeed, the linear restrictions $\mu_{i}^{M} \geq \mu_{i} \geq \mu_{i}^{m}$, $\sum_{i} \mu_{i}=1$ describe a bounded polytope with a finite number of vertices [19].

Well-known linear-programming-related methods to obtain the membership vector vertices may be used (related to the obtention of the basic feasible solutions in an LP problem [19]). Details are omitted for brevity. 


\section{Local stability analysis}

By using the transformed models discussed in the previous section, local stability results may be obtained by the lemmas in Section 2 .

Lemma 2 The polynomial region $\Omega^{*} \subset \Omega$

$$
\Omega^{*}=\left\{x: V(x) \leq V_{M}, V(x)>0\right\}
$$

is a basin of attraction of the equilibrium point $x=0$ of the system (3) if

$$
V_{M} \leq \min \{V(x): x \in \partial \Omega\}
$$

where $\partial \Omega$ denotes the boundary of $\Omega$ and $V(x)$ verifies:

$$
-\frac{d V}{d x} p_{k}(Z)^{*} \in \Sigma_{x} \quad k: 1, \ldots, n_{v}
$$

i.e., all trajectories with initial state in $\Omega^{*}$ converge assymptotically to $x=0$.

Proof: As, by Lemma 1, the system can be expressed in $\Omega$ as:

$$
\dot{x}=\sum_{k=1}^{n_{v}} \beta_{k}(z) p_{k}(Z)^{*}
$$

if the SOS condition (19) is feasible for a positive polynomial $V(x)$, then a Lyapunov function proving stability is founded.

As the expression of the local system (8) is not valid outside $\Omega$, then the local stability can only be proved in the largest polynomial region $\Omega^{*}$ contained in $\Omega$, which will be defined by a value of $V_{M}$ equal to the minimum value of $V(x)$ in the boundary of $\Omega$ $(\partial \Omega)$.

The following theorem is useful in order to set up a SOS characterisation of the largest polynomial region in $\Omega$ which is a Lyapunov equipotential.

In this work, we will search for "well-conditioned" Lyapunov functions so that large spherical balls around $x=0$ can be proved to belong to the domain of attraction. In particular, we will seek that every level set of the Lyapunov function, $V(x)=V_{M}$, is included between two spheres, one inner sphere of radius $\left(\lambda^{-1} V_{M}\right)^{1 / 2}$ and one outher sphere of radius $V_{M}^{1 / 2}$.

Suppose $\Omega$ defined as a symmetric polytope that contains $x=0$ :

$$
\Omega=\left\{x:\left|a_{i}^{T} x\right| \leq 1 \quad i: 1, \ldots, n_{k}\right\}
$$

and $\epsilon(x)=\sum_{i} x_{i}^{2}$.

Theorem 1 Consider the system (3) and a symmetric polytopic region $\Omega$. $V(x)$ is a Lyapunov function and its level set $V(x)=V_{M}$ is included between the two spheres considered in the above paragraph if the following SOS problem:

$$
x^{T} \lambda x-V(x)+\varphi_{i}\left(\left(a_{i}^{T} x\right)^{2}-1\right) \in \Sigma_{x} i: 1 \ldots n_{p}
$$

$$
\begin{gathered}
V(x)-\epsilon(x)+v_{j}\left(\left(a_{i}^{T} x\right)^{2}-1\right) \in \Sigma_{x} j: 1 \ldots n_{p} \\
-\frac{d V}{d x} p_{k}(Z)^{*}+\psi_{l}\left(\left(a_{i}^{T} x\right)^{2}-1\right) \in \Sigma_{x} \begin{array}{l}
l: 1 \ldots n_{p} \\
k: 1 \ldots n_{v}
\end{array}
\end{gathered}
$$

is feasible, where $\lambda$ is a spherical size parameter and $\Omega$ is defined as (20). The largest valid polynomial region (contained in $\Omega$ ) $\Theta=\left\{x: V(x) \leq V_{M}\right\}$ found, is the basin of attraction of $x=0$ proved.

Proof: The information of the polytope $\Omega$ added with positive SOS multipliers $\varphi_{i}, v_{j}$ and $\psi_{l}$, keep $V(x)$ being a valid Lyapunov function inside $\Omega$, by Lemma 2. And the conditions (21) and (22) are the two spherical inclusion conditions needed.

Iterative Algorithm. The results in previous sections may be combined in order to obtain an algorithm to compute an estimate of the largest ball around $x=0$ for which attraction is ensured, by means of obtaining the "roundest" Lyapunov function via trying to close the "gap" between the outer sphere of radius $V_{M}^{1 / 2}$ and the inner radius $\left(\lambda^{-1} V_{M}\right)^{1 / 2}$.

Basically, the procedure will first check the extreme cases; (1) checking for feasibility of SOS problems as stated in Section 2, (2) checking for stability of the linearised model around $x=0$.

Then, an exploration must be made solving the following SOS problem, checking different region sizes in order to obtain the maximum sphere:

minimize $\lambda$ subject to

$$
\begin{array}{rlll}
x^{T} \lambda x-V(x)+\varphi_{i}\left(\left(a_{i}^{T} x\right)^{2}-1\right) & \in & \Sigma_{x} & i: 1 \ldots n_{p} \\
V(x)-\epsilon(x)+v_{j}\left(\left(a_{i}^{T} x\right)^{2}-1\right) & \in & \Sigma_{x} & j: 1 \ldots n_{p} \\
-\frac{d V}{d x} p_{k}(Z)^{*}+\psi_{l}\left(\left(a_{i}^{T} x\right)^{2}-1\right) & \in & \Sigma_{x} l: 1 \ldots n_{p} \\
& & & k: 1 \ldots n_{v}
\end{array}
$$

So, for Theorem 1 along with the SOS objective, this procedure maximize the radius of the quadratically invariant sphere contained in $\Omega$. Indeed, such a maximum-size sphere may appear at an intermediate point, as example will later show. Hence, no bisection or related idea is applicable in principle as the radius provable with the above theorem sometimes increases when increasing the domain of interest and some others decreases, with local maxima and minima.

\section{Example}

This section presents an example in order to clarify the procedure. The evolution of the radius of the largest guaranteed sphere is shown as the size of the region of interest is increased. The estimated basin of attraction shape is also shown increasing the region of interest. 


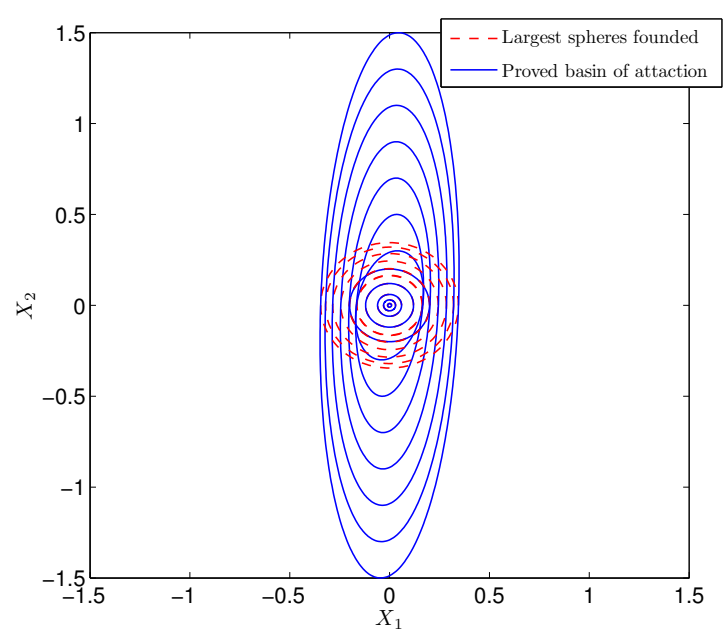

Figure 1: Basins of attraction for different sizes of (square) operating domain.

Let us have a two rules fuzzy polynomial system model, with its corresponding membership functions, given next:

$$
\begin{gathered}
\dot{x}=\sum_{i=1}^{2} \mu_{i}(x) p_{i}(x) \\
\mu_{1}(x)=e^{-\left(x_{1}^{2}+x_{2}^{2}\right)} ; \mu_{2}(x)=1-\mu_{1}(x) \\
p_{1}=\left(\begin{array}{c}
-0.116 x_{1}+0.015 x_{2}^{4}+0.0603 x_{2} \\
0.2 x_{1}^{2} x_{2}-0.0603 x_{1}-0.4711 x_{2}
\end{array}\right) \\
p_{2}=\left(\begin{array}{c}
-0.1106 x_{1}+0.03 x_{2}^{4}-0.2796 x_{2} \\
-0.1 x_{1}^{2} x_{2}+5.8714 x_{1}-0.4763 x_{2}
\end{array}\right)
\end{gathered}
$$

Define $\Omega_{k}$ as a square region in the plane $\left(x_{1}, x_{2}\right)$ given by:

$$
\Omega_{k}=\left\{x||\left(01 / \rho_{k}\right) x|\leq 1,|\left(1 / \rho_{k} 0\right) x \mid \leq 1\right\}
$$

where $k$ is the grid-point (iteration) number, and $\rho_{k}$ is a size parameter to be changed in the iterations.

Notice that the minimum and maximum values of $\mu_{i}$ in $\Omega$ are easily obtained in the center and the extreme vertex of the square. Indeed, in this case, the monotonicity of the memberships implies that their maximum and minimum are easily computable.

The procedures in previous sections allow determining the largest sphere around $\mathrm{x}=0$ for which local polynomial stability holds. In this case we took quadratic stability for simplicity.

Let us consider for the fist iteration $\rho_{1}=0.01$ and next iterations every 0.01 increments until reaching infeasibility.

The maximum sphere local stability conditions are obtained by solving the SOS in Theorem 1 for each region $\Omega_{k}$, replacing (23) by:

$$
\begin{aligned}
& -2\left[x_{1} x_{2}\right] P\left(\mu_{1 m} p_{1}+\mu_{2 m} p_{2}\right)+ \\
& \psi_{1}\left(\left(\frac{x_{1}}{\rho}\right)^{2}-1\right)+\psi_{2}\left(\left(\frac{x_{2}}{\rho}\right)^{2}-1\right)=\Sigma_{x}
\end{aligned}
$$

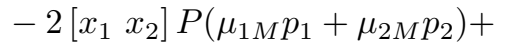

$$
\begin{aligned}
& \psi_{1}\left(\left(\frac{x_{1}}{\rho}\right)^{2}-1\right)+\psi_{2}\left(\left(\frac{x_{2}}{\rho}\right)^{2}-1\right)=\Sigma_{x}
\end{aligned}
$$

where $\mu_{1 m}, \mu_{2 m}$ are calculated in the center of square $(x=0)$, and $\mu_{1 M}, \mu_{2 M}$ in the extreme $\operatorname{vertex}\left(\left[x_{1}, x_{2}\right]=[\rho, \rho]\right)$.

The ellipsoids and largest sphere contained on it are shown in Figure 1 for some of the tested values. Note how the ill-conditioned ellipsoids encompass a much smaller area than the square in which the Lyapunov function decreases, and that fact is even more accentuated when looking at the spheres: for instance, with a square of size 0.6 , the largest stable sphere has a radius 0.18 , i.e., its area is only $7 \%$ of that of the square in which the fuzzy model is valid.

Now, if decay rate stability is also checked for this system, only is necessary to add

$$
-2 \alpha\left[x_{1} x_{2}\right] P\left[x_{1} x_{2}\right]^{T}
$$

to the conditions $(26),(27)$. Then, starting with $\alpha=$ 0 , by increasing it the SOS conditions become more restrictive so the provable radius will be smaller. Figure 2 shows the evolutions of radius with $\Omega$ for different decay rates. The upper plot corresponds to plain stability $(\alpha=0)$.

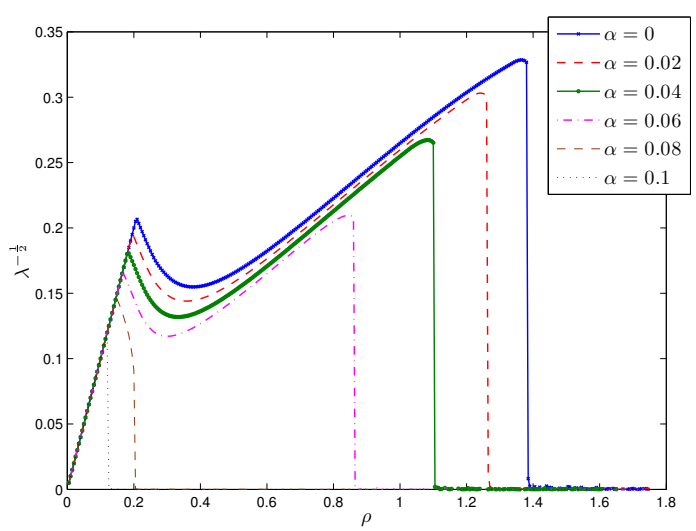

Figure 2: Decay rate radius evolution

It can be shown that there is no solution in all tested regions $\Omega_{k}$, and as we approach the unfeasibility point, the obtained radius gets quickly very small. Also the evolution of the maximum radius proved is not linear so iterations are needed to find the best basin of attraction .

\section{Conclusions}

This paper has discussed some issues on local stability and control design in a polynomial framework with the objective to find the largest sphere which is guaranteed to belong to the domain of attraction 
of an original nonlinear system. A polytopic expression of the memberships functions in a reduced domain of attraction is the basic idea. The maximum provable radius is a non-monotonic function of the size of the region of interest which requires exploration to be found.

\section{References}

[1] Kazuo Tanaka and Hua O. Wang. Fuzzy control systems design and analysis: a linear matrix inequality approach. Wiley-Interscience publication. John Wiley and Sons, 2 edition, 2001.

[2] A. Sala and C. Ariño. Polynomial fuzzy models for nonlinear control: A Taylor series approach. Fuzzy Systems, IEEE Transactions on, 17:1284-1295, August 2009.

[3] Stephen Boyd, Laurent El Ghaoui, Eric Feron, and Venkataramanan Balakrishnan. Linear matrix inequalities in system and control theory. Number 15 in SIAM studies in applied mathematics. SIAM, 1994.

[4] H.O Wang, K. Tanaka, and M.F. Griffin. An approach to fuzzy control of nonlinear systems: stability anddesign issues. Fuzzy Systems, IEEE Transactions on, 4:14-23, February 1996.

[5] S. Prajna, A. Papachristodoulou, P. Seiler, and P.A. Parrilo. SOSTOOLS: control applications and new developments. Computer Aided Control Systems Design, 2004 IEEE International Symposium on, pages 315 - 320, September 2004.

[6] S. Prajna, A. Papachristodoulou, and Fen Wu. Nonlinear control synthesis by sum of squares optimization: a Lyapunov-based approach. Control Conference, 2004. 5th Asian, 1:157 - 165, July 2004.

[7] T. Takagi and M. Sugeno. Fuzzy identification of systems and its applications to modeling and control. IEEE transactions on systems, man, and cybernetics, 15(1):116-132, February 1985.

[8] K. Tanaka, T. Hori, and HO Wang. A multiple Lyapunov function approach to stabilization of fuzzy control systems. IEEE Transactions on Fuzzy Systems, 11(4):582-589, 2003.
[9] T.M. Guerra and L. Vermeiren. LMI-based relaxed nonquadratic stabilization conditions for nonlinear systems in the Takagi-Sugeno's form. Automatica, 40:823-829, May 2004.

[10] A. Sala and C. Ariño. Local Stability Of OpenAnd Closed-Loop Fuzzy Systems. In IEEE International Symposium on Intelligent Control (ISIC'2006), pages 2384-2389, Munich (Alemania), 2006. IEEE.

[11] A. Sala. Reducing the gap between fuzzy and nonlinear control (invited talk). Proc. 3rd IFAC Workshop Adv. Fuzzy-Neural Control, Valenciennes, France [Online], pages 1-6, 2007.

[12] S. Prajna, A. Papachristodoulou, P. Seiler, and P. A. Parrilo. SOSTOOLS: Sum of squares optimization toolbox for MATLAB, 2004.

[13] K.C. Toh, M.J. Todd, and R.H. Tütüncü. SDPT3 - a MATLAB software package for semidefinite programming., October 1998.

[14] S. Prajna, A. Papachristodoulou, and P.A. Parrilo. Introducing SOSTOOLS: a general purpose sum of squares programming solver. Decision and Control, 2002, Proceedings of the 41st IEEE Conference on, 1:741 - 746 vol.1, dec. 2002.

[15] K. Tanaka, H. Yoshida, H. Ohtake, and H. O. Wang. A sum of squares approach to stability analysis of polynomial fuzzy systems. Proc. Amer. Control Conf, pages 4071-4076, 2007.

[16] M. Johansson, A. Rantzer, and K.-E. Arzen. Piecewise quadratic stability of fuzzy systems. Fuzzy Systems, IEEE Transactions on, 7:713722, December 1999.

[17] de Oliveira M.C., Bernussou J., and Geromel J.C. A new discrete-time robust stability condition. Systems and Control Letters, 37(4):261265, July 1999.

[18] J.L. Pitarch, C.V. Ariño, F. Bedate, and A. Sala. Local fuzzy modeling: Maximising the basin of attraction. In Fuzzy Systems (FUZZ), 2010 IEEE International Conference on, pages $1-7$, july 2010.

[19] D.G. Luenberger. Linear and Nonlinear Programming. Springer, 2 edition, 2003. 